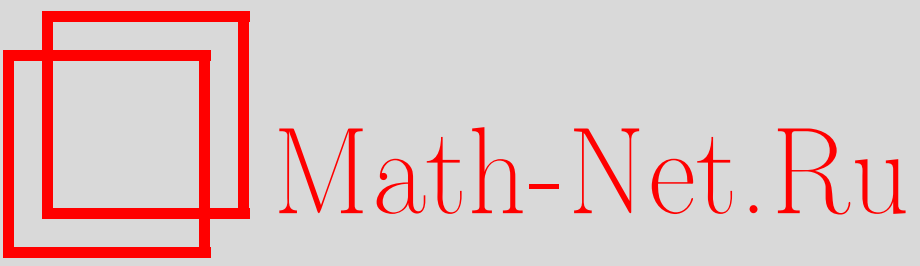

А. Ф. Заусаев, Л. А. Соловьев, Применение метода регуляризации к дифференциальным уравнениям движения комет, Вестн. Сам. гос. техн. ун-та. Сер. Физ.-мат. науки, 2009, выпуск 2(), 288-292

DOI: https://doi.org/10.14498/vsgtu710

Использование Общероссийского математического портала Math-Net.Ru подразумевает, что вы прочитали и согласны с пользовательским соглашением

http://www. mathnet.ru/rus/agreement

Параметры загрузки:

IP : 3.80 .181 .102

26 апреля 2023 г., $17: 02: 46$ 
Небесная механика и астрономия

УДК 523.642

\section{ПРИМЕНЕНИЕ МЕТОДА РЕГУЛЯРИЗАЦИИ К ДИФФЕРЕНЦИАЛЬНЫМ УРАВНЕНИЯМ ДВИЖЕНИЯ КОМЕТ}

\section{А. Ф. Заусаев, Л. А. Соловъев}

Самарский государственный технический университет, 443100, Самара, ул. Молодогвардейская, 244.

E-mail: Zausaev_AF@mail.ru

Применен метод регуляризации к дифференииалъньм уравнениям двиэения комет. Проведено численное интегрирование уравнений движения десяти короткопериодических комет на интервале времени с 1800 по 2204 гг. с использованием оскулирующих элементов больших планет. Показана высокая эфбективность данного метода для короткопериодических комет.

Ключевые слова: интерполяиия, численное интегрирование, дифференииальные уравнения движения, метод оскулирующих элементов.

Дифференциальные уравнения движения кометы в гелиоцентрической системе координат с учётом релятивистских эффектов от Солнца имеют следующий вид [1]:

$$
\begin{aligned}
\frac{d^{2} X}{d t^{2}}= & -k^{2}(1+m) \frac{X}{r^{3}}+\sum_{i} k^{2} m_{i}\left(\frac{X_{i}-X}{\Delta_{i}^{3}}-\frac{X_{i}}{r_{i}^{3}}\right)+ \\
& +\frac{k^{2}}{c^{2}}\left[(4-2 \alpha) \frac{k^{2}}{r^{4}} X-(1+\alpha) \frac{\dot{r}^{2}}{r^{3}} X+3 \alpha \frac{(X \dot{X})^{2}}{r^{5}} X+(4-2 \alpha) \frac{(X \dot{X})}{r^{3}} \dot{X}\right]
\end{aligned}
$$

где $X$-матрица-столбец с элементами $x, y, z ; X_{i}$-матрица-столбец с элементами $x_{i}, y_{i}, z_{i} ; m, x, y, z$ - масса и гелиоцентрические координаты возмущаемого тела; $m_{i}, x_{i}, y_{i}, z_{i}$ - массы и гелиоцентрические координаты больших планет; $r, \Delta_{i}$, $r_{i}$ - расстояния, вычисляемые по следующим формулам: $r^{2}=x^{2}+y^{2}+z^{2}, \Delta_{i}^{2}=$ $=\left(x_{i}-x\right)^{2}+\left(y_{i}-y\right)^{2}+\left(z_{i}-z\right)^{2}, r_{i}^{2}=x_{i}^{2}+y_{i}^{2}+z_{i}^{2} ; \dot{X}$ - матрица-столбец с элементами $\dot{x}, \dot{y}, \dot{z} ; k$ - постоянная Гаусса; $c$-скорость света; $\alpha$ - параметр, характеризующий выбор системы координат. Случай $\alpha=1$ соответствует стандартным координатам, случай $\alpha=0$ - гармоническим координатам.

Система дифференциальных уравнений (1) описывает движение $n$ материальных точек в гелиоцентрической системе координат. Решение этой системы существенно упрощается, если известны координаты возмущающих тел, так как в этом случае вместо задачи $n$ тел решается задача двух тел. Уравнения (1) сингулярны в начале координат. Для кометных орбит, имеющих большие эксцентриситеты, изменение расстояния от центрального тела приводит к неравномерному изменению правых частей дифференциальных уравнений (1). При численном интегрировании такая неравномерность требует постоянного изменения шага интегрирования, что приводит к потере точности вычислений. Преодолеть эти трудности можно путём преобразования сингулярных дифференциальных уравнений (1) в регулярные.

Анатолий Федорович Заусаев (д.ф.-м.н.), профессор, каф. прикладной математики и информатики. Леонид Александрович Соловъев, аспирант, каф. прикладной математики и информатики. 
Процедуру, позволяющую устранить в дифференциальных уравнениях движения сингулярность, называют регуляризацией. Регуляризацию выполняют в два этапа. Первый этап состоит в том, чтобы получить регулярные функции, описывающие движение, а также в устранении бесконечного возрастания скорости в точке соударения. Это достигается путём замены физического времени $t$ на фиктивное время $s$, с помощью преобразования

$$
\frac{d t}{d s}=r
$$

Если использовать фиктивное время в качестве независимой переменной, то уравнение движения (1) примет вид

$$
\begin{aligned}
r X^{\prime \prime}- & r^{\prime} X^{\prime}+k^{2}(1+m) X=r^{3} \sum_{i} k^{2} m_{i}\left(\frac{X_{i}-X}{\Delta_{i}^{3}}-\frac{X_{i}}{r_{i}^{3}}\right)+ \\
+ & \frac{k^{2}}{c^{2}}\left[(4-2 \alpha) \frac{k^{2}}{r} X-(1+\alpha) \frac{r^{\prime 2}}{r^{2}} X+3 \alpha \frac{\left(X X^{\prime}\right)^{2}}{r^{4}} X+(4-2 \alpha) \frac{\left(X X^{\prime}\right)}{r^{2}} X^{\prime}\right],
\end{aligned}
$$

где штрих указывает на дифференцирование по $s$.

В дифференциальные уравнения движения (3) входят координаты возмущающих планет $x_{i}, y_{i}, z_{i}$. Эффективность работы программы при решении уравнений (3) можно повысить путём использования банка данных координат больших планет.

При численном интегрировании уравнений движения (3) необходимо вычислять физическое время на каждом шаге интегрирования с той же точностью, с которой вычисляются координаты возмущаемого тела. Выполнения данного требования можно достичь следующим образом. Продифференцировав уравнение $(2)$ по $s$, получим:

$$
t^{\prime \prime}=r^{\prime}
$$

Совместным решением уравнений (3) и (4) можно получить требуемую точность для физического времени по известному фиктивному времени.

Численное интегрирование системы дифференциальных уравнений (3) и (4) для десяти короткопериодических комет проводилось методом Эверхарта 27-го порядка с использованием банка данных оскулирующих элементов больших планет [3]. Интегрирование уравнений движения проводилось двумя методами. В первом случае решались уравнения движения, полученные в работе [4], в которых учтены как гравитационные, так и релятивистские эффекты. Данные об эволюции орбит этих комет содержатся в работе [5]. Во втором случае численное интегрирование уравнений (3) и (4) проводилось с учётом первого этапа регуляризации с использованием оскулирующих элементов.

В табл. 1, 2 приведены элементы орбит, полученные в результате интегрирования первым и вторым способом, обозначены соответственно «Совм. интегр.» и «Регул. интегр.»; $\Delta S$ - абсолютная величина разности в значениях орбитальных элементов, полученных первым и вторым методами. Начальные данные элементов орбит комет взяты из каталога [5].

Как видно из табл. 1 и 2, максимальные расхождения в элементах кометных орбит для всех 10 комет в обоих методах на конце интервала интегрирования незначительны. При этом в угловых элементах максимальное расхождение наблюдается в средней аномалии у кометы D/Biela $\left(0,0057^{\circ}\right)$, а отличия в остальных элементах орбит не превышают точности оптических наблюдений. Так как оба метода дают незначительные расхождения в элементах орбит, то это позволяет считать, что метод регуляризации с использованием оскулирующих элементов можно применять для исследования эволюции орбит короткопериодических комет на интервале времени с 1800 по 2204 гг. 
Т аблица 1

Элементы орбит комет, полученные совместным интегрированием уравнений движения, а также с учётом регуляризации ( $\mathrm{T}=18000105)$

\begin{tabular}{|c|c|c|c|c|c|c|}
\hline & $M$ & $a$ & $e$ & $\omega$ & $\Omega$ & $i$ \\
\hline \multicolumn{7}{|c|}{$\mathrm{P} /$ Halley } \\
\hline Совм. интегр. & 191,4946 & 18,052023 & 0,968665 & 111,3587 & 58,0169 & 162,2889 \\
\hline Регул. интегр. & 191,4944 & 18,052032 & 0,968665 & 111,3586 & 58,0168 & 162,2889 \\
\hline$\Delta S$ & 0,0002 & $9,00 \mathrm{E}-06$ & 0 & $1,00 \mathrm{E}-04$ & $1,00 \mathrm{E}-04$ & 0 \\
\hline \multicolumn{7}{|c|}{$\mathrm{P} /$ Encke } \\
\hline Совм. интегр. & 60,5893 & 2,216543 & 0,845846 & 182,3158 & 337,1369 & 13,567 \\
\hline Регул. интегр. & 60,5894 & 2,216543 & 0,845846 & 182,3158 & 337,1369 & 13,567 \\
\hline$\Delta S$ & 0,0001 & 0 & 0 & 0 & 0 & 0 \\
\hline \multicolumn{7}{|c|}{$\mathrm{D} /$ Biela } \\
\hline Совм. интегр. & 37,7136 & 3,559049 & 0,746668 & 217,8642 & 254,2752 & 13,6419 \\
\hline Регул. интегр. & 37,7135 & 3,559049 & 0,746668 & 217,8642 & 254,2752 & 13,6419 \\
\hline$\Delta S$ & $1,00 \mathrm{E}-04$ & 0 & 0 & 0 & 0 & 0 \\
\hline \multicolumn{7}{|c|}{$\mathrm{P} /$ Faye } \\
\hline Совм. интегр. & 73,4076 & 3,866193 & 0,556092 & 191,2737 & 226,7653 & 7,3485 \\
\hline Регул. интегр. & 73,4029 & 3,866174 & 0,556095 & 191,2729 & 226,7657 & 7,3483 \\
\hline$\Delta S$ & 0,0047 & 0,000019 & $3,00 \mathrm{E}-06$ & $8,00 \mathrm{E}-04$ & $4,00 \mathrm{E}-04$ & $2,00 \mathrm{E}-04$ \\
\hline \multicolumn{7}{|c|}{ D/Brorsen } \\
\hline Совм. интегр. & 63,0994 & 3,027727 & 0,733766 & 7,5765 & 106,8219 & 45,287 \\
\hline Регул. интегр. & 63,09939 & 3,027759 & 0,733772 & 7,5767 & 106,8219 & 45,2868 \\
\hline$\Delta S$ & $1,00 \mathrm{E}-05$ & $3,20 \mathrm{E}-05$ & $6,00 \mathrm{E}-06$ & 0,0002 & 0 & 0,0002 \\
\hline \multicolumn{7}{|c|}{$\mathrm{P} / \mathrm{d}^{\prime}$ Arrest } \\
\hline Совм. интегр. & 37,2982 & 3,413185 & 0,669816 & 170,2287 & 153,3499 & 13,0505 \\
\hline Регул. интегр. & 37,2989 & 3,413184 & 0,669816 & 170,2285 & 153,35 & 13,0505 \\
\hline$\Delta S$ & 0,0007 & $1,00 \mathrm{E}-06$ & 0 & 0,0002 & 0,0001 & 0 \\
\hline \multicolumn{7}{|c|}{ P/Pons-Winnecke } \\
\hline Совм. интегр. & 208,1477 & 3,337746 & 0,72313 & 5,6173 & 268,8229 & 2,6807 \\
\hline Регул. интегр. & 208,1477 & 3,337746 & 0,72313 & 5,6169 & 268,8235 & 2,6808 \\
\hline$\Delta S$ & 0 & 0 & 0 & 0,0004 & 0,0006 & $1,00 \mathrm{E}-04$ \\
\hline \multicolumn{7}{|c|}{$\mathrm{P} /$ Tuttle } \\
\hline Совм. интегр. & 260,0457 & 5,748858 & 0,819734 & 206,8318 & 271,662 & 54,3911 \\
\hline Регул. интегр. & 260,0452 & 5,748858 & 0,819734 & 206,8318 & 271,662 & 54,3911 \\
\hline$\Delta S$ & 0,0005 & 0 & 0 & 0 & 0 & 0 \\
\hline \multicolumn{7}{|c|}{$\mathrm{P} /$ Tempel 1} \\
\hline Совм. интегр. & 280,1281 & 3,128444 & 0,524341 & 131,945 & 105,2602 & 6,2927 \\
\hline Регул. интегр. & 280,1292 & 3,128442 & 0,524342 & 131,9436 & 105,2616 & 6,2925 \\
\hline$\Delta S$ & 0,001 & 2,00E-06 & $1,00 \mathrm{E}-06$ & 0,0014 & 0,0014 & 0,0002 \\
\hline \multicolumn{7}{|c|}{$\mathrm{P} /$ Tempel 2} \\
\hline Совм. интегр. & 86,4843 & 3,023543 & 0,546203 & 178,2391 & 125,7648 & 12,4545 \\
\hline Регул. интегр. & 86,4834 & 3,023543 & 0,546202 & 178,2392 & 125,7648 & 12,4545 \\
\hline$\Delta S$ & 0,0009 & 0 & $1,00 \mathrm{E}-06$ & 0,0001 & 0 & 0 \\
\hline
\end{tabular}


Т аблица 2

Элементы орбит комет, полученные совместным интегрированием уравнений движения, а также с учётом регуляризации ( $\mathrm{T}=220412$ 13)

\begin{tabular}{|c|c|c|c|c|c|c|}
\hline & $\bar{M}$ & $a$ & $e$ & $\omega$ & $\Omega$ & $i$ \\
\hline \multicolumn{7}{|c|}{$\overline{\mathrm{P} / \text { Halley }}$} \\
\hline Совм. интегр. & 340,7337 & 17,82704 & 0,967167 & 115,0549 & 62,4152 & 161,7191 \\
\hline Регул. интегр. & 340,737 & 17,82697 & 0,967167 & 115,055 & 62,4152 & 161,7191 \\
\hline$\Delta S$ & 0,0033 & $7,00 \mathrm{E}-05$ & 0 & 0,0001 & 0 & 0 \\
\hline \multicolumn{7}{|c|}{$\mathrm{P} /$ Encke } \\
\hline Совм. интегр. & 316,0063 & 2,221484 & 0,847531 & 192,2903 & 330,3659 & 9,2604 \\
\hline Регул. интегр. & 316,0035 & 2,221485 & 0,847531 & 192,2903 & 330,3659 & 9,2604 \\
\hline$\Delta S$ & 0,0028 & $1,00 \mathrm{E}-06$ & 0 & 0 & 0 & 0 \\
\hline \multicolumn{7}{|c|}{$\mathrm{D} /$ Biela } \\
\hline Совм. интегр. & 155,9316 & 3,527373 & 0,763495 & 326,0849 & 139,6366 & 14,611 \\
\hline Регул. интегр. & 155,9259 & 3,527377 & 0,763496 & 326,0847 & 139,6368 & 14,6105 \\
\hline$\Delta S$ & 0,0057 & $4,00 \mathrm{E}-06$ & $1,00 \mathrm{E}-06$ & 0,0002 & 0,0002 & 0,0005 \\
\hline \multicolumn{7}{|c|}{$\mathrm{P} /$ Faye } \\
\hline Совм. интегр. & 281,7301 & 3,71421 & 0,610773 & 220,5747 & 167,8499 & 5,098 \\
\hline Регул. интегр. & 281,7299 & 3,714211 & 0,610772 & 220,5747 & 167,85 & 5,098 \\
\hline$\Delta S$ & 0,0003 & $1,00 \mathrm{E}-06$ & 1,00E-06 & 0 & 0,0001 & 0 \\
\hline \multicolumn{7}{|c|}{ D/Brorsen } \\
\hline Совм. интегр. & 41,2152 & 3,073071 & 0,863266 & 73,7416 & 39,4331 & 6,7586 \\
\hline Регул. интегр. & 41,2165 & 3,073068 & 0,863267 & 73,7376 & 39,4371 & 6,7588 \\
\hline$\Delta S$ & 0,0013 & $3,00 \mathrm{E}-06$ & 1,00E-06 & 0,004 & 0,004 & 0,0002 \\
\hline \multicolumn{7}{|c|}{$\mathrm{P} / \mathrm{d}^{\prime}$ Arrest } \\
\hline Совм. интегр. & 81,8749 & 3,789775 & 0,492992 & 169,5791 & 128,5464 & 23,3064 \\
\hline Регул. интегр. & 81,8754 & 3,789774 & 0,492993 & 169,5791 & 128,5465 & 23,3063 \\
\hline$\Delta S$ & 0,0005 & $1,00 \mathrm{E}-06$ & $1,00 \mathrm{E}-06$ & 0 & 0,0001 & 0,0001 \\
\hline \multicolumn{7}{|c|}{ P/Pons-Winnecke } \\
\hline Совм. интегр. & 305,1878 & 3,366807 & 0,672255 & 193,3037 & 79,3204 & 18,0484 \\
\hline Регул. интегр. & 305,1877 & 3,366807 & 0,672255 & 193,3037 & 79,3204 & 18,0484 \\
\hline$\Delta S$ & 0,0001 & 0 & 0 & 0 & 0 & 0 \\
\hline \multicolumn{7}{|c|}{$\mathrm{P} /$ Tuttle } \\
\hline Совм. интегр. & 146,0471 & 5,698912 & 0,822694 & 207,7192 & 269,2077 & 55,0828 \\
\hline Регул. интегр. & 146,0471 & 5,698912 & 0,822694 & 207,7192 & 269,2077 & 55,0828 \\
\hline$\Delta S$ & 0 & 0 & 0 & 0 & 0 & 0 \\
\hline \multicolumn{7}{|c|}{$\mathrm{P} /$ Tempel 1} \\
\hline Совм. интегр. & 274,3968 & 3,223767 & 0,498254 & 200,3509 & 56,1666 & 8,2521 \\
\hline Регул. интегр. & 274,3972 & 3,223768 & 0,498253 & 200,351 & 56,1665 & 8,2521 \\
\hline$\Delta S$ & 0,0004 & $1,00 \mathrm{E}-06$ & 1,00E-06 & 0,0001 & 0,0001 & 0 \\
\hline \multicolumn{7}{|c|}{$\mathrm{P} /$ Tempel 2} \\
\hline Совм. интегр. & 2,8662 & 3,027102 & 0,569752 & 217,0771 & 102,824 & 8,8981 \\
\hline Регул. интегр. & 2,8647 & 3,027103 & 0,569751 & 217,0773 & 102,8239 & 8,898 \\
\hline$\Delta S$ & 0,0015 & $1,00 \mathrm{E}-06$ & 1,00E-06 & 0,0002 & 0,0001 & 0,0001 \\
\hline
\end{tabular}


Следует также отметить, что по быстродействию метод регуляризации с использованием оскулирующих элементов почти в 5 раз превосходит метод совместного интегрирования.

На основании выше изложенного можно сделать вывод, что при исследовании эволюции орбит как короткопериодических, так и долгопериодических комет с большими эксцентриситетами предпочтительнее проводить решение уравнений (3) и (4) с использованием оскулирующих элементов по сравнению с методом совместного интегрирования.

Работа выполнена в рамках АВЦП «Развитие научного потенииала высшей школы (2009-2010)» (проект РНП 2.1.1/745).

\section{БИБЛИОГРАФИЧЕСКИЙ СПИСОК}

1. Брумберг В. А. Релятивистская небесная механика. - М.: Наука, 1972. - 382 с.

2. Штифель E., Шейфеле Г. Линейная и регулярная небесная механика. - М.: Наука, 1975. $-304 \mathrm{c}$

3. Заусаев А.Ф., Заусаев Д.А. Численное интегрирование уравнений движения малых тел Солнечной системы с использованием оскулирующих элементов больших планет / В сб.: Труды Шестой Всероссийской научной конференции с международным участием (1-4 июня 2009 г.). Часть 3: Дифференциальные уравнения и краевые задачи / Матем. моделирование и краев. задачи. - Самара: СамГТУ, 2009. - С. 125-130.

4. Newhall X. X., Standish E. M., Williams J. G. DE 102 - A numerically integrated ephemeris of the moon and planets spanning forty-four centuries // Astron. Astrophys, 1983. - Vol. 125, No. 1. - P. $150-167$.

5. Заусаев A. Ф., Заусаев A.А. Каталог орбитальной эволюции короткопериодических комет с 1800 по 2204 гг. - М.: Машиностроение-1, 2007. - 410 с.

Поступила в редакцию 20/VII.2009; в окончательном варианте $-10 / \mathrm{IX} / 2009$.

MSC: $70 F 15,70-08$

\section{APPLICATION OF THE REGULARIZATION METHOD TO DIFFERENTIAL EQUATIONS OF COMETS' MOTION}

\section{A. F. Zausaev, L. A. Solov'ev}

Samara State Technical University,

244, Molodogvardeyskaya str., Samara, 443100.

E-mail: Zausaev_AF@mail.ru

Regularization method for differential equation of comets' motion is used. Numerical integration of the equation of motion of 10 short-period comets during the period of 1800 to 2204 with the use of osculating elements of the large planets is done. The high efficiency of this method for short-period comets study is demonstrated. efficiency of this method for short-period comets is shown.

Key words: numerical integration, differential equation of motion, method of the osculation elements.

Original article submitted 20/VII.2009; revision submitted 10/IX/2009.

Anatoliy F. Zausaev (Dr. Sci. (Phys. \& Math.)), Professor, Dept. of Applied Mathematics \& Computer Science. Leonid A. Solov'ev, Postgraduate Student, Dept. of Applied Mathematics \& Computer Science. 